\title{
Closed-Loop Control of Vortex Shedding on a Two-Dimensional Flat-Plate Airfoil at a Low Reynolds Number*
}

\author{
Won Tae Joe, ${ }^{\dagger}$ Kunihiko Taira, ${ }^{\ddagger}$ Tim Colonius, ${ }^{\S}$ Douglas G. MacMynowski $₫$ \\ California Institute of Technology, Pasadena, CA, 91125 \\ Gilead Tadmor ${ }^{\|}$ \\ Northeastern University, Boston, MA, 02115
}

\begin{abstract}
Open- and closed-loop control of vortex shedding in two-dimensional flow over a flat plate at high angles of attack is numerically investigated at a Reynolds number of 300 . Unsteady actuation is modeled as a body force near the leading or trailing edge, and is directed either upstream or downstream. For moderate angles of attack, sinusoidal forcing at the natural shedding frequency results in phase locking, with a periodic variation of lift at the same frequency. However, at sufficiently high angles of attack, subharmonics of the forcing frequency are also excited and the average lift over the forcing period varies from cycle to cycle in a complex manner. It is observed that the periods with the highest averaged lift are associated with particular phase difference between the forcing and the lift. We design a feedback algorithm to lock the forcing with the phase shift associated with the highest period-averaged lift. It is shown that the compensator results in a stable phaselocked limit cycle for a larger range of forcing frequencies than the open-loop control, and that it is able to stabilize otherwise unstable high-lift limit cycles that cannot be obtained with open-loop control. For example at an angle of attack of $40^{\circ}$, the feedback controller can increase the averaged lift coefficient from 1.35 to 2.43 , an increase of $80 \%$.
\end{abstract}

\section{Introduction}

It has been observed in flapping flight of insects and birds, that a leading-edge vortex (LEV) is formed and stays stably attached during most of the wing's downstroke, and detaches only when the wing reverses its direction. ${ }^{1,2}$ This results in greatly enhanced lift when the wing is at high angle of attack to the flow. Motivated by the aerodynamic benefits associated with stable LEV, the present research seeks to use unsteady blowing near the leading and/or trailing edges of a translating wing in order to control the formation and detachment of the LEV. The simple model problem described here is part of a larger research program ${ }^{3}$ that includes three-dimensional flow simulations, ${ }^{4}$ experiments, ${ }^{5}$ and reduced-order modeling. ${ }^{6}$

Unsteady actuation has been used in the past for separation control. ${ }^{7,8}$ Several studies have demonstrated that unsteady actuation near the separation point can reattach the flow, shorten the separation bubble, or suppress vortex shedding to reduce drag. ${ }^{8-10}$ The purpose of our study is not to control the separation, but rather to control the formation and shedding of vortical structures near the leading or trailing edges in order to utilize the unsteady vortical forces (both lift and drag) they produce.

As a step towards understanding control in a broader context, we consider here a highly idealized model problem of two-dimensional flow over a flat plate at high angle of attack. The natural flow exhibits a periodic

\footnotetext{
*Work supported by a Multidisciplinary Research Initiative from the United States Air Force Office of Scientific Research (FA9550-05-1-0369, Program Manager: Dr. Fariba Fahroo).

${ }^{\dagger}$ Ph.D. Candidate, Mechanical Engineering, Student Member AIAA

$\ddagger$ Ph.D. Candidate, Mechanical Engineering, Student Member AIAA

$\S$ Professor, Mechanical Engineering, Member AIAA

ISenior Research Fellow, Control and Dynamical Systems, Member AIAA

$\|$ Professor, Electrical and Computer Engineering, Member AIAA
} 
vortex shedding that leads to very large fluctuating lift and drag. We model actuators as simple unsteady body forces placed near the leading or trailing edges, directed either upstream or downstream to the flow. Open-loop control with periodic pulsing at the natural shedding frequency is first investigated for various actuator configurations over a range of post-stall angles of attack.

In certain cases, primarily for lower angles of attack, open-loop forcing results in a phase-locked limit cycle with lift varying at the frequency of actuation. For sufficiently high angles of attack, however, subharmonic frequencies are excited and a more complex limit cycle behavior is obtained. The period-averaged lift varies from cycle to cycle, and it is observed that higher lift is associated with a particular phase shift between the forcing and the lift. Particularly for upstream blowing at the trailing edge, during certain cycles the periodaveraged lift can exceed the maximum lift achieved during the natural shedding cycle. In order to phase lock the forcing to the particular phase shift associated with the highest period-averaged lift, a feedback algorithm is designed and its behavior is explored in detail at $\alpha=30^{\circ}$ and $40^{\circ}$, where $\alpha$ denotes the angle of attack. It is shown that the feedback compensator results in stable phase-locked limit cycles for a larger range of forcing frequencies than the open-loop control, and it can stabilize high-lift limit cycles that are otherwise unstable with open-loop control.

\section{Numerical Method}

Simulations of flow over a two-dimensional flat plate at $R e=300$ are performed with the immersed boundary projection method combined with a multi-domain technique. ${ }^{11,12}$ This method is capable of solving for incompressible flows over an arbitrary-shaped body in motion and deformation. Here we employ this method with the flat plate being stationary. In what follows, all velocities and length scales are nondimensionalized by the freestream velocity and the chord, $U_{\infty}$ and $c$, respectively.

A grid size of $250 \times 150$ is used on the most resolved grid domain of $[-1,4] \times[-1.5,1.5]$ in the streamwise $(x)$ and vertical $(y)$ directions centered around the plate, and a timestep of $\Delta t=0.004$ is used. This domain is stretched onto multiple larger domains up to $[-8,32] \times[12,12]$ to minimize the effect of the outflow boundary condition on the near flowfield around the flat plate. ${ }^{12}$ Selected cases were run on grids with finer resolution and larger extents to demonstrate sufficient convergence of the results presented.

The lift coefficient on the flat plate is defined by

$$
C_{L}=\frac{F_{y}}{\frac{1}{2} \rho U_{\infty}^{2} c},
$$

where $F_{y}$ is the lift on the plate and $\rho$ is the freestream density of the fluid. Since the force obtained is normal to the plate and $F_{y}$ is only the vertical component of the normal force, the increase of the normal force increases both the lift and drag. As the angle of attack increases, the drag component of the normal force is increased while the lift component is reduced. For high angles of attack, this might result in decrease of the lift-to-drag ratio even in the presence of lift enhancement. However, for the purpose of demonstrating the control algorithm to achieve high lift, we will only consider the lift component of the normal force and only report $C_{L}$.

In practice, actuators produce a jet-like flow that can lead to complex spatial and temporal characteristics. However, for the purpose of investigating the control of shedding, we model the actuation as a point body force smeared over a few grid points and define its strength by specifying its velocity, $U_{\mathrm{j}}$. In defining the momentum injection added by the forcing, the width of the actuator is estimated as the grid spacing, $\Delta x$. The momentum coefficient, defined in Eq. (2), is the ratio between the momentum injected by the forcing and that of the freestream.

$$
C_{\mu}=\frac{\rho \bar{U}_{\mathrm{j}}^{2} \Delta x}{\frac{1}{2} \rho U_{\infty}^{2} c}
$$

The values of $C_{\mu}$ reported are based on the average jet velocity, $\bar{U}_{\mathrm{j}}$, fixed at 0.5 , and the width of the actuator, $\Delta x=0.02$. This corresponds to a fixed $C_{\mu}$ of 0.01 for all of the cases considered here. For each actuation location, two cases of blowing angles are considered, one directed downstream and the other directed upstream as illustrated in figure 1. 


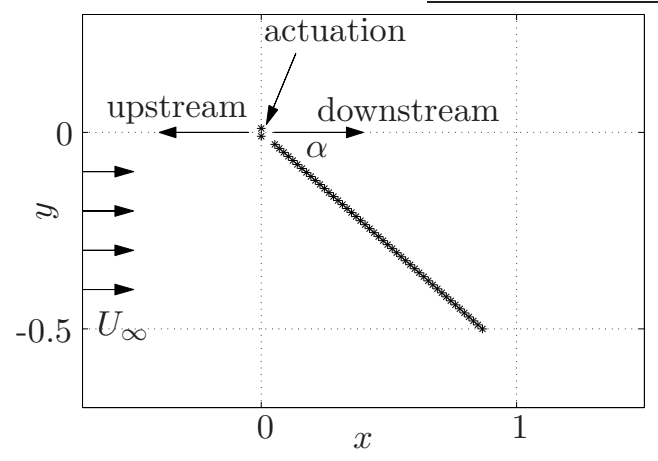

(a)

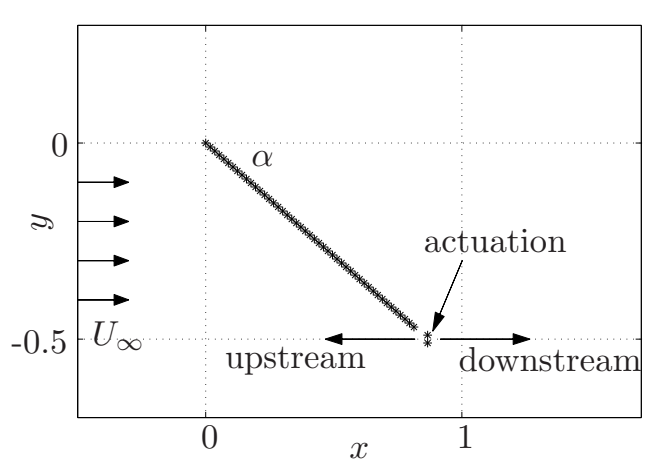

(b)

Figure 1. Schematic of actuation at (a)leading-edge and (b)trailing-edge

\section{Results}

\section{III.A. Uncontrolled Flow}

For the translating flat plate at $R e=300$, steady attached flow is observed for $\alpha<10^{\circ}$. At $\alpha=10^{\circ}$, the flow is observed to be separated but remains steady. The flow undergoes a Hopf bifurcation between angles of attack of $12^{\circ}$ and $15^{\circ},{ }^{3}$ after which vortex shedding occurs with natural shedding frequency, $\omega_{\mathrm{n}}$ $\left(\omega_{\mathrm{n}} \in[3.65,1.39]\right.$ for $\left.\alpha \in\left[15^{\circ}, 50^{\circ}\right]\right)$. Using the vertical projection of the airfoil to the freestream, we find that $\omega_{\mathrm{n}}$ can be nicely scaled, for $\alpha \geq 30^{\circ}$, to a Strouhal number of $S t=f_{\mathrm{n}} c \sin (\alpha) / U_{\infty} \approx 0.2$, where $f_{\mathrm{n}}=\omega_{\mathrm{n}} /(2 \pi)$. This agrees with the wake Strouhal number for vortex shedding behind two-dimensional bluff bodies. ${ }^{13-15}$ The unsteady shedding cycle consists of vortices of opposite sign alternately shed from the leading and trailing edges, respectively, creating periodic oscillations in the lift and drag. As $\alpha$ is increased, larger vortex structures are formed, inducing a larger amplitude of oscillation in the force exerted on the plate. For $\alpha \geq 30^{\circ}$, the vortex structure on the suction side of the plate is observed to be created from the leading edge and can be viewed as a transient LEV, or, equivalently, a dynamic stall vortex (DSV) that occurs during a rapid pitch up. Maximum lift is found when the LEV is brought down to the suction side of the plate as it grows in strength. The lift decreases as the new vortex structure of the opposite sign is formed at the trailing edge. This trailing-edge vortex (TEV) pushes up the LEV sitting on the suction side of the plate, and finally halts its growth causing it to pinch-off and shed into the wake.

\section{III.B. Open-loop control}

In order to investigate the effect of unsteady blowing on these vortex shedding cycles, we first consider open-loop control of periodic pulsing with different blowing angles at the leading and trailing edge of the plate. The nondimensional jet velocity is set as $U_{\mathrm{j}}=\bar{U}_{\mathrm{j}}+U_{\mathrm{j}}^{\prime} \sin \left(\omega_{\mathrm{f}} t\right)$, where $\bar{U}_{\mathrm{j}}=0.5$ and $U_{\mathrm{j}}^{\prime}=0.5$. Since this study is focused on maximizing lift from shedding of the coherent vortex structures rather than the suppression of shedding or separation, $\omega_{\mathrm{f}}$ is initially chosen to be the natural shedding frequency for each $\alpha$, to which the unsteady shedding of the large coherent vortex structure will likely be amplified the most. ${ }^{16,17}$ In the next two sections we examine leading and trailing edge actuation, respectively, in each case forcing the flow at the natural shedding frequency $\left(\omega_{\mathrm{f}}=\omega_{\mathrm{n}}\right)$, unless otherwise noted.

\section{III.B.1. Leading-edge actuation}

Figure 2 shows the lift coefficient with actuation at the leading edge directed downstream (left) and upstream (right). In each figure, the uncontrolled flow (baseline) is overlaid in grey. The dashed lines show the minimum and maximum of the lift signal whose overall average is shown in the circles in between. For cases where the lift is not phase locked to the forcing signal, the period-averaged lift (averaged over each actuation period) is also plotted in pink to indicate a different average lift over each period.

Blowing downstream provides extra momentum at the leading-edge in addition to that of the freestream. This amplifies the unsteady shedding of vortex structures, resulting in larger magnitudes of the lift fluctuations. The forced flow exhibits higher maximum lift but also lower minimum lift, below that of the baseline 
flow. As a result, blowing downstream does not significantly benefit the overall average lift.

However, when the actuation is directed upstream, the resulting amplification of the unsteady shedding has a more positive effect on the average lift. For $\alpha<25^{\circ}$, the flow locks onto the forcing $2 \sim 3$ periods after the actuation is initiated. However at higher $\alpha$, the flow fails to lock onto the forcing frequency and displays a more complicated limit cycle. Subharmonics of the forcing frequency are also excited. The lift signal over time in figure 3(a) displays an example of this subharmonic behavior at $\alpha=50^{\circ}$ where each subharmonic limit cycle consists of several periods with a different period-averaged lift. Figure 3(b) shows the lift as a function of the jet velocity, and shows that the actuation produces the highest lift when $U_{\mathrm{j}}$ is in phase with the $C_{L}$ (maximum $C_{L}$ when $U_{\mathrm{j}}$ is maximum). However, the succeeding period becomes slightly out of phase and the lift decreases. Each period within the subharmonic limit cycle is observed to be associated with a particular phase shift, $\phi$, between the forcing signal and the lift, yielding a particular period-averaged lift. The actuation period associated with the highest average lift is plotted in a thicker line. At each $\alpha$, there is a particular $\phi$, resulting in the highest average lift over an actuation period. If the feedback allows us to accordingly adjust the frequency of actuation to phase lock the flow at these $\phi$, then we could repeatedly produce the highest average lift period. This feedback design will be revisited later.

It might be counter-intuitive that upstream actuation at the leading edge achieves such a lift enhancement and performs better than downstream actuation. However, experiments at Reynolds number of the order of $3 \times 10^{5}$ by Rullan et al. ${ }^{18}$ demonstrated that unsteady blowing upstream, parallel to the chord at the leadingedge of a sharp-edged, circular arc airfoil at various $\alpha$ beyond stall leads to averaged pressure distribution that resulted in higher lift than that of the baseline flow. They achieved lift increase as high as $30 \%$ with momentum coefficient of $C_{\mu}{ }^{\prime}=C_{\mu} / \sin (\alpha) \approx 1 \%$, scaled with the vertical projection of the airfoil and the actuation pulsating at the shedding frequency of the airfoil.

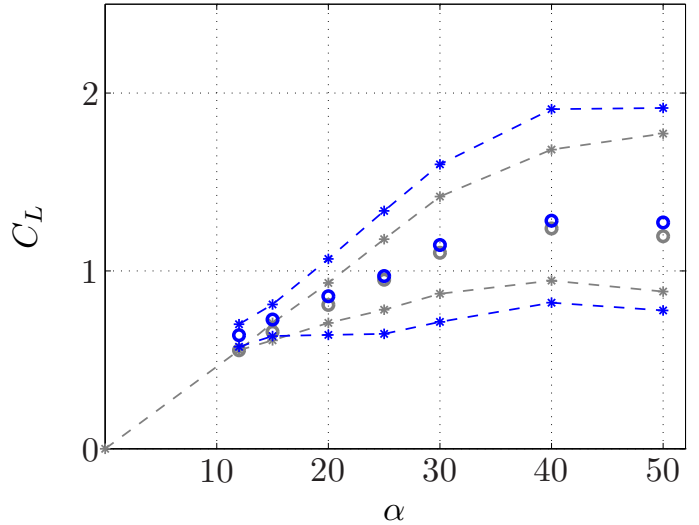

(a) downstream

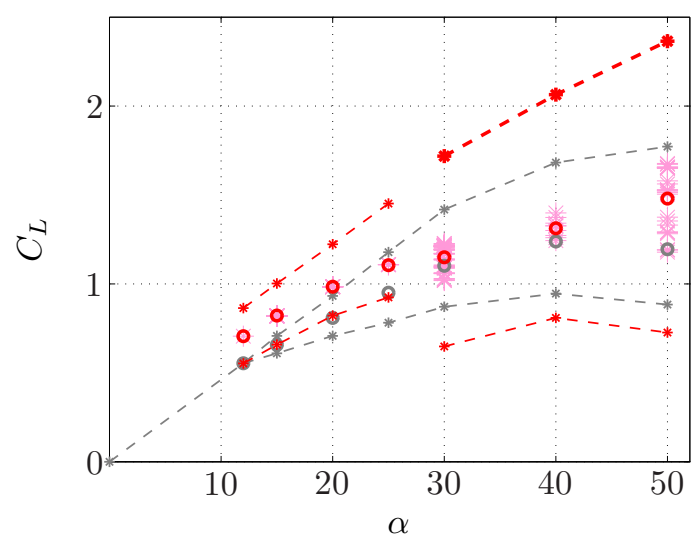

(b) upstream

Figure 2. Leading-edge actuation: maximum and minimum lift $(*, *, *)$ and its average over time $(\bigcirc, \bigcirc, \bigcirc)$ for baseline (grey), and downstream (blue) and upstream (red) actuation. Actuation is applied at the natural shedding frequency, $\omega_{f}=\omega_{n}$. For cases where the flow is not phase locked to the forcing signal, period-averaged lift over each actuation period is plotted $(*)$ to indicate a subharmonic limit cycle.

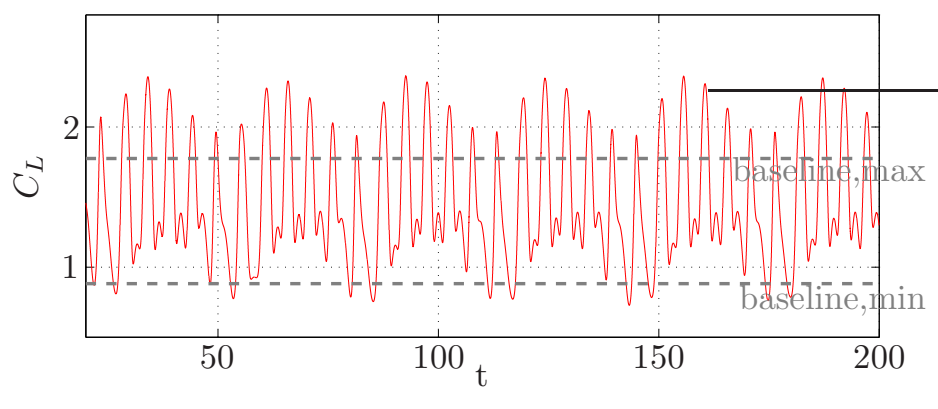

(a)

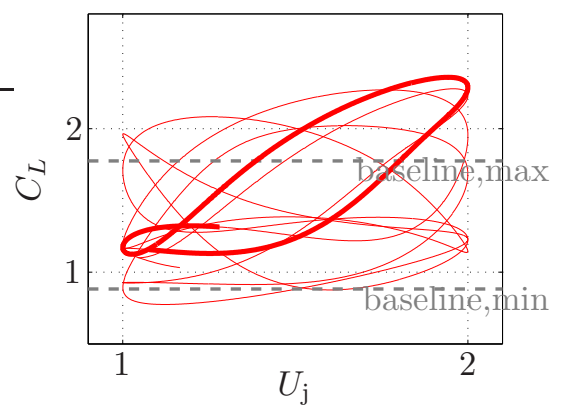

(b)

Figure 3. Lift with upstream actuation at the leading edge at the natural shedding frequency $\left(\omega_{f}=\omega_{n}\right)$ for $\alpha=50^{\circ}$. 


\section{III.B.2. Trailing-edge actuation}

In figure 4, the lift performance of the open-loop actuation at the natural shedding frequency at the trailing edge is investigated in a similar manner as in figure 2. Blowing downstream exerts a negative effect on the average lift, yielding a lower minimum lift than that of the baseline flow with a similar maximum lift. However, when the forcing is directed upstream, the forced flow displays a significant lift enhancement. The forcing excites the vortex shedding cycle even for $\alpha$ below the Hopf bifurcation. For $\alpha \leq 15^{\circ}$, the flow locks onto the forcing after $2 \sim 3$ periods. However, for $\alpha \geq 20^{\circ}$, the subharmonic resonance is excited. This is similar to the observation with upstream blowing at the leading edge, but the subharmonic resonance is excited at a lower $\alpha$ for the trailing-edge actuation than that for the leading-edge actuation.

Each period within the subharmonic limit cycle is again observed to be associated with a particular $\phi$, resulting in a particular period-averaged lift for each period. We denote the $\phi$ associated with the highest period-averaged lift at each $\alpha$ as $\phi_{\text {best. }}$. Particularly at $\alpha=30^{\circ}$ and $40^{\circ}$, $\phi_{\text {best }}$ was observed to be approximately -0.257 and -0.116 radians, respectively. For trailing-edge actuation, the period-averaged lift at high $\alpha$ is, in many cases, greater than the maximum lift occurring in the baseline flow. This suggests a greater potential for the trailing-edge feedback actuation to sustain the flow with the highest period-averaged lift. Consequently, we would obtain a phase-locked flow that has an average lift as high as the maximum lift of the baseline flow (or even higher).

In general, blowing upstream at both the leading and trailing edge creates significant enhancement in the average lift. However, blowing upstream at the trailing edge provides larger increase in lift than that of the leading-edge actuation. These findings are similar to observations made by Huang et al. ${ }^{19}$ who investigated the effect of blowing and suction control at various locations on the upper surface of a NACA0012 airfoil.

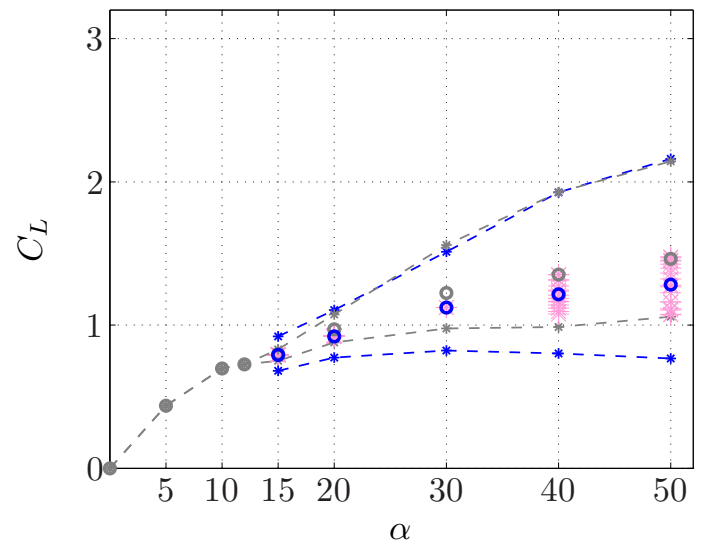

(a) downstream



(b) upstream

Figure 4. Trailing-edge actuation: see Figure 2 for a description.

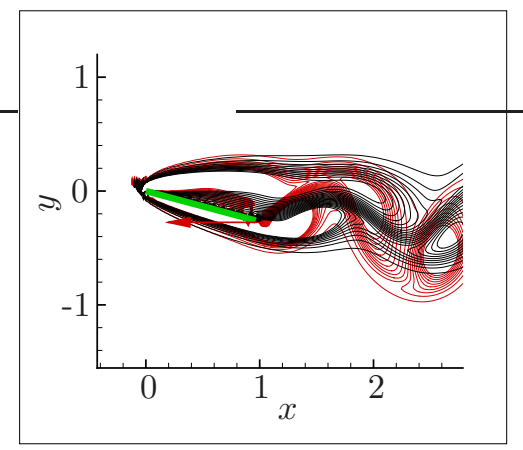

(a) $\alpha=15^{\circ}$

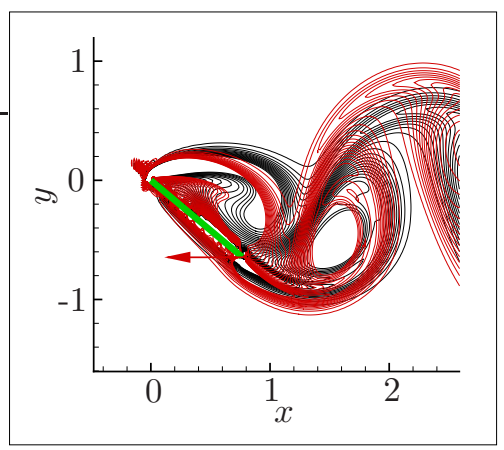

(b) $\alpha=40^{\circ}$

Figure 5. Vorticity contour at the time of maximum lift for baseline (black) and upstream actuation (red) at the trailing edge at the natural shedding frequency $\left(\omega_{f}=\omega_{n}\right)$. 


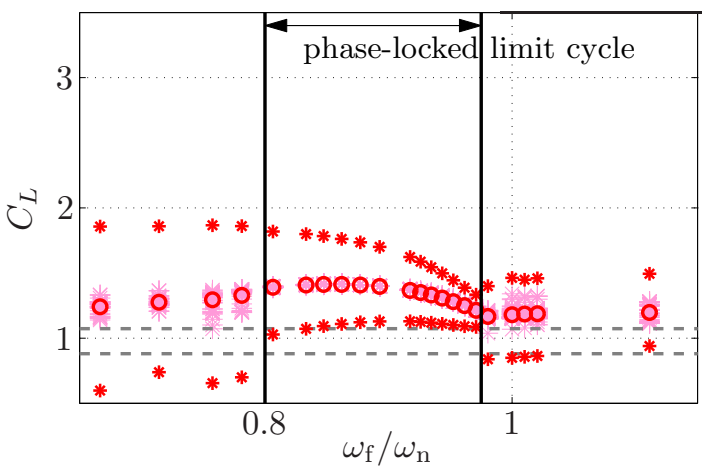

(a) $\alpha=20^{\circ}$

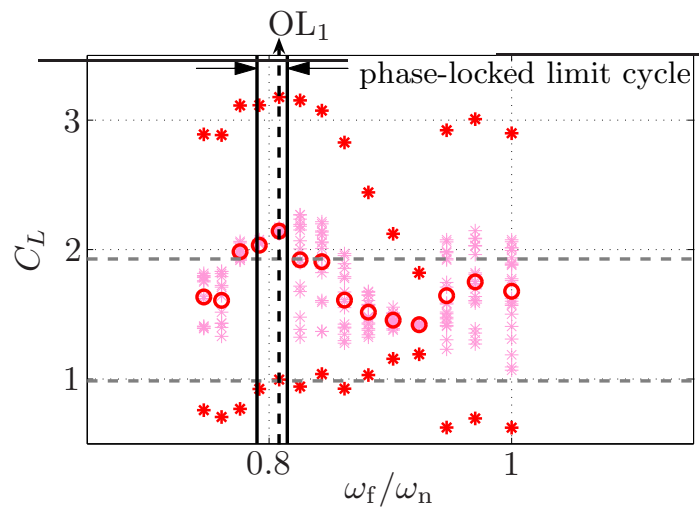

(c) $\alpha=40^{\circ}$

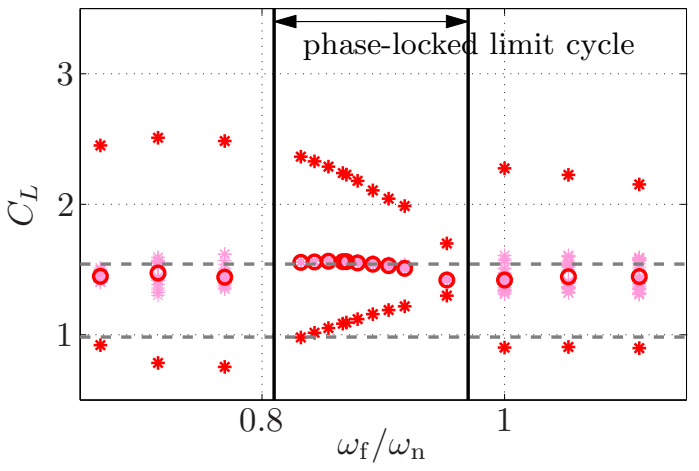

(b) $\alpha=30^{\circ}$

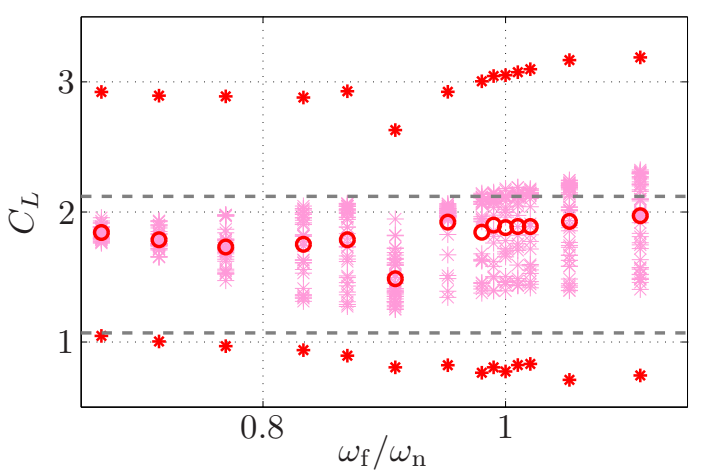

(d) $\alpha=50^{\circ}$

Figure 6. Trailing-edge actuation: maximum and minimum lift $(*)$, average lift $(O)$, and period-averaged lift (*) over a range of open-loop forcing frequency, $\omega_{\mathrm{f}}$. Maximum and minimum lift of baseline (- - -) case is shown as a reference

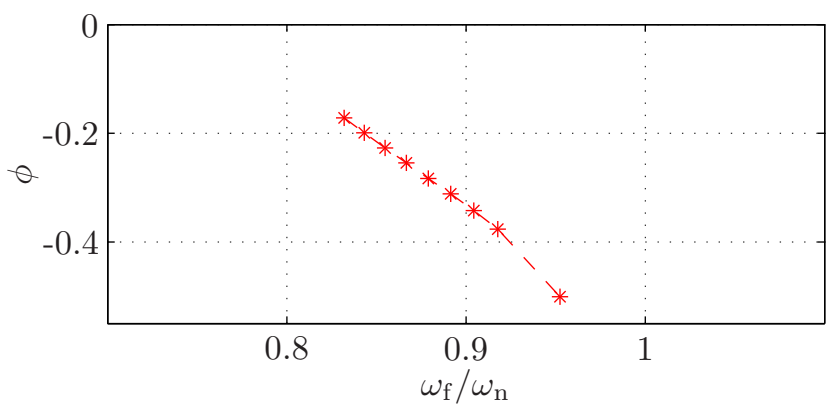

Figure 7. Trailing-edge actuation: phase shift of the forcing signal, $U_{j}$, relative to the lift signal, $C_{L}$, for phase-locked flows, over a range of open-loop forcing frequency, $\omega_{\mathrm{f}}\left(\alpha=30^{\circ}\right)$.

They considered steady blowing and suction at $R e=5 \times 10^{5}$ and $\alpha=18^{\circ}$ and demonstrated that blowing at the leading edge directed downstream exerts a negative effect, decreasing lift and increasing drag at the same time, but suction increases lift by creating a larger and lower pressure zone on the airfoil's upper surface. They also observed that the actuation near the trailing-edge on the upper surface, $0.8 c$ from the leading edge, improves lift and drag characteristics by manipulating the circulation of the TEV.

In order to understand the lift-enhancing mechanism of upstream actuation at the trailing edge, we compare the vorticity contours at the time of maximum lift for the cases of baseline and upstream actuation at the trailing edge, for $\alpha=15^{\circ}$ and $40^{\circ}$ in figure 5. Actuation feeds extra circulation to the TEV which induces a stronger downwash near the trailing edge. As a result, vortex structure on the suction side is pulled down closer to the plate and the backflow near the trailing edge is reduced. Particularly at $\alpha=40^{\circ}$, this delays the interference of the newly forming TEV with the LEV residing on the suction side. It also lengthens the duration over which the vortex structure is formed from the leading edge. These results also agree with the observations that the period associated with the highest lift within a subharmonic cycle in 
figure 4(b) has a longer period than that of the baseline flow. This might indicate that there exists a forcing frequency below $\omega_{\mathrm{n}}$, at which the flow becomes phase locked to the forcing at a higher lift than that of the baseline flow.

Thus, we next investigate the possibility of the existence of shedding cycles that are phase locked to the open-loop forcing signal. Figure 6 shows the lift response to the variation in open-loop forcing frequency for $20^{\circ} \leq \alpha \leq 50^{\circ}$, above which upstream actuation at $\omega_{\mathrm{f}}=\omega_{\mathrm{n}}$ fails to phase lock the flow.

Over a range of frequency below $\omega_{\mathrm{n}}$, the flow is phase locked to the actuation with its average lift near the maximum period-averaged lift of the flow actuated at $\omega_{\mathrm{f}}=\omega_{\mathrm{n}}$. As we go deeper into stall by increasing $\alpha$, the domain of attraction for the phase-locked limit cycle decreases, and finally at $\alpha=50^{\circ}$, actuation failed to phase lock the flow over the range of forcing frequencies considered.

Figure 7 shows the corresponding phase shift, $\phi$, over this range of $\omega_{\mathrm{f}}$ that achieves a phase-locked flow. Recall that the subharmonic cycle (excited with upstream blowing at $\omega_{\mathrm{n}}$ ) consists of several actuation periods with a distinct $\phi$ associated with a particular period-averaged lift. Also, its highest period-averaged lift is associated with $\phi=\phi_{\text {best }} \approx-0.257$. As $\omega_{\mathrm{f}}$ is decreased below $\omega_{\mathrm{n}}$, the flow phase locks onto one of those periods observed in the subharmonic limit cycle at $\alpha=30^{\circ}$. As $\omega_{\mathrm{f}}$ is decreased further, the flow is phase locked onto different periods with different $\phi$, closer to $\phi_{\text {best }}$ with higher average lift. Finally, at $\omega_{\mathrm{f}} / \omega_{\mathrm{n}}=0.867$, the actuation is able to lock the flow at the best period achieved with forcing at $\omega_{\mathrm{n}}$. This indicates that each phase-locked limit cycle of the vortex shedding could be characterized by its frequency and the phase shift, yielding a particular maximum, minimum, and average lift.

If the feedback allows us to adjust the frequency of the actuation accordingly to keep the phase shift between the forcing signal and the lift constant (for example at $\phi=\phi_{\text {best }}$ ), we might be able to reproduce the high-lift shedding cycles over a wide range of $\alpha$. Thus as an attempt to achieve the desired phase-locked shedding cycle, we feedback lift into the controller, whose details are described in the next section.

\section{III.C. Closed-loop control}

Open-loop periodic forcing can lead to limit cycles with a high average lift, but with a decreasing domain of attraction as $\alpha$ increases. Our goal with closed-loop control is to obtain forced limit cycles with the maximum average lift. This may involve increasing the range of the forcing frequencies for the phase-locked limit cycles, or stabilizing limit cycles that would not be stable without feedback.

Since the actuated flows with the highest average lift seem to be characterized by a distinct phase shift of the forcing relative to the lift at each $\alpha$, we feedback $C_{L}$ in an attempt to phase lock the flow at these high-lift states. Direct feedback of $C_{L}$ with appropriate gain would only allow us to force the flow to be in-phase with $U_{\mathrm{j}}$. However, since the observed best phase shifts between $C_{L}$ and $U_{\mathrm{j}}$ are negative, shifting direct feedback signal requires us to know the frequency of the forced flow a priori. Instead, we assume that the lift signal being fed back is approximately sinusoidal. In such cases lift can be expressed as:

$$
\begin{aligned}
C_{L}(t) & =a_{0}+A_{L} \cos \left(\omega_{\mathrm{i}} t+\theta\right), \\
C_{L}(t) & =a_{0}+a_{1} \cos \left(\omega_{\mathrm{i}} t\right)+b_{1} \sin \left(\omega_{\mathrm{i}} t\right), \\
a_{1} & =A_{L} \cos (\theta), \\
b_{1} & =A_{L} \sin (\theta) .
\end{aligned}
$$

Assuming that $A_{L}$ and $\theta$ are slowly varying in time, we can estimate $a_{1}$ and $b_{1}$ to be the Fourier mode over a moving window:

$$
\begin{aligned}
a_{1}(t) & =\frac{2}{T_{i}} \int_{t-T_{i}}^{t} L\left(t^{\prime}\right) \cos \left(\omega_{\mathrm{i}} t^{\prime}\right) d t^{\prime}, \\
b_{1}(t) & =\frac{2}{T_{i}} \int_{t-T_{i}}^{t} L\left(t^{\prime}\right) \sin \left(\omega_{\mathrm{i}} t^{\prime}\right) d t^{\prime}, \\
\omega_{i} & =\frac{2 \pi}{T_{i}} .
\end{aligned}
$$

Then we feedback a phase-shifted version of this demodulated lift signal as the jet velocity, $U_{\mathrm{j}}$ with appropriate gain, $K_{p}$ :

$$
U_{\mathrm{j}}(t)=a_{0}+K_{p}\left(a_{1}(t) \cos \left(\omega_{\mathrm{i}} t+\phi_{\mathrm{i}}\right)+b_{1}(t) \sin \left(\omega_{\mathrm{i}} t+\phi_{\mathrm{i}}\right)\right)
$$


where $a_{0}$ is the average value of the output $U_{\mathrm{j}}$, which can be prescribed as 0.5 to fix $C_{\mu}=0.01$. We also adjust $K_{p}$ periodically, such that the rms amplitude of $U_{\mathrm{j}}$ remains steady and similar to that of open-loop control, i.e. $U_{\mathrm{j}}$ varies from 0 to 1 .

The configuration of our feedback control is shown in figure 8. Lift is fed back to the controller which has two parameters: demodulation frequency, $\omega_{\mathrm{i}}$, and the desired phase shift $\phi_{\mathrm{i}}$, which we fix to be the $\phi_{\text {best }}$ estimated from the open-loop forcing simulations ( $\phi_{\mathrm{i}} \approx-0.257$ and -0.116 for $\alpha=30^{\circ}$ and $40^{\circ}$, respectively). The controller outputs a sinusoid that is phase shifted relative to the dominant frequency of the lift signal, as $U_{\mathrm{j}}$ to the flow. The flow system outputs $C_{L}$, which has a frequency $\omega_{\mathrm{o}}$ and a phase shift $\phi_{\mathrm{o}}$ relative to the input signal $U_{\mathrm{j}}$.

Note that $\omega_{\mathrm{i}}$ is chosen preferably close to the dominant output frequency of the forced flow, $\omega_{\mathrm{o}}$. However in practice, they will not always be equal to each other, and in that case, $\phi_{\mathrm{o}}$ will be different from $\phi_{\mathrm{i}}$. Also, if $C_{L}$ is phase-locked to $U_{\mathrm{j}}$, the frequency of $U_{\mathrm{j}}$ will always be the same as the frequency of $C_{L}$, but the phase shift between them, $\phi_{\mathrm{o}}$ is not necessarily equal to $\phi_{\mathrm{i}}$ (unless $\omega_{\mathrm{o}}=\omega_{\mathrm{i}}$ in which case $\phi_{\mathrm{o}}=\phi_{\mathrm{i}}$ ). Thus, rather than fixing the phase shift (and therefore allowing the frequency content to be determined only by the flow), the current feedback controller parameterizes a family of closed-loop flow behaviors with frequency and phase shift characteristics determined by the combination of the controller and flow dynamics.

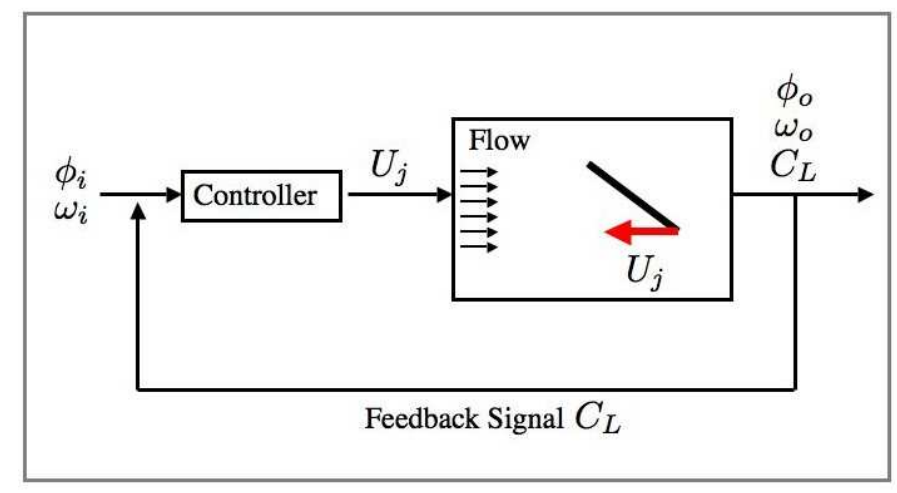

Figure 8. Feedback control configuration.

In figure 9, we investigate how this feedback algorithm behaves over a wide range of $\omega_{\mathrm{i}}$ with a fixed $\phi_{\mathrm{i}}=\phi_{\text {best }}$, for $\alpha=30^{\circ}$ and $40^{\circ}$. Only the ranges of $\omega_{i}$ that the feedback controller successfully phase locks the flow are plotted $\left(\omega_{i} \in(0.65,1.25)\right.$ for $\alpha=30^{\circ}$ and $\omega_{i} \in(0.7,1.2)$ for $\left.\alpha=40^{\circ}\right)$. Since $\omega_{\mathrm{i}}$ is only the internal tuning parameter of this feedback compensator, each limit cycle we obtain is characterized by its output frequency, $\omega_{\mathrm{o}}$, and the corresponding $\phi_{\mathrm{o}}$, which determine the lift behavior. Note that over a wide range of $\omega_{\mathrm{i}}$, output frequency only varies between 0.8 and 0.95 . However, $\phi_{\mathrm{o}}$ changes sufficiently to yield different lift performance.

At $\alpha=30^{\circ}$, we compare figure 9(a) to the corresponding lift and phase shift characteristics of phaselocked limit cycles over various open-loop forcing frequencies in figure $6(\mathrm{~b})$ and figure 7 . At each $\omega_{\mathrm{i}} / \omega_{\mathrm{n}}$ higher than 0.8 , the feedback locks the flow at the limit cycle with $\omega_{\mathrm{o}}$ and $\phi_{\mathrm{o}}$ similar to the frequency and $\phi$ of a phase-locked limit cycle achieved by the open-loop forcing at $0.8<\omega_{\mathrm{f}} / \omega_{\mathrm{n}}<0.95$. For example, the limit cycle that the feedback phase locked at $\omega_{\mathrm{o}} / \omega_{\mathrm{n}} \approx 0.86$ with $\phi_{\mathrm{o}} \approx-0.25$ has the similar lift behavior (minimum, maximum, and average lift) to that of the limit cycle phase locked to the open-loop forcing at $\omega_{\mathrm{f}} / \omega_{\mathrm{n}} \approx 0.86$ and $\phi \approx-0.25$. In this case, they can be considered as the same limit cycle. Thus, for $\omega_{\mathrm{i}} / \omega_{\mathrm{n}}>0.8$, the feedback achieves the same phase-locked limit cycles also obtained by the open-loop forcing at $0.8<\omega_{\mathrm{f}} / \omega_{\mathrm{n}}<0.95$. However, for $\omega_{\mathrm{i}} / \omega_{\mathrm{n}} \approx 0.7$, we observe that the resulting limit cycle is phase locked to the feedback forcing at $\omega_{\mathrm{o}} / \omega_{\mathrm{n}} \approx 0.85$ but with the phase shifts that are different from that of limit cycle phase locked to open-loop forcing at $\omega_{\mathrm{f}} \approx 0.85$. Also, it is interesting to note that the feedback achieved several limit cycles that are phase locked at the similar frequency $\left(\omega_{\mathrm{o}} / \omega_{\mathrm{n}} \approx 0.8\right)$ but with different $\phi_{\mathrm{o}}$, resulting in different lift behavior (whereas open-loop control can only achieve one limit cycle at a given forcing frequency). Thus, the feedback results in phase-locked limit cycles that are not attainable by the open-loop forcing. 


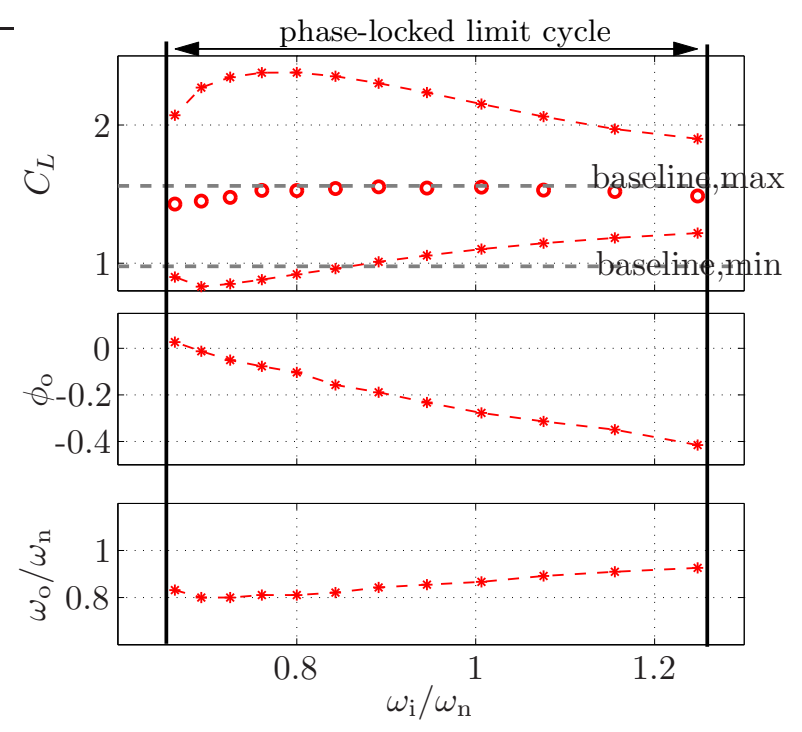

(a) $\alpha=30^{\circ}$

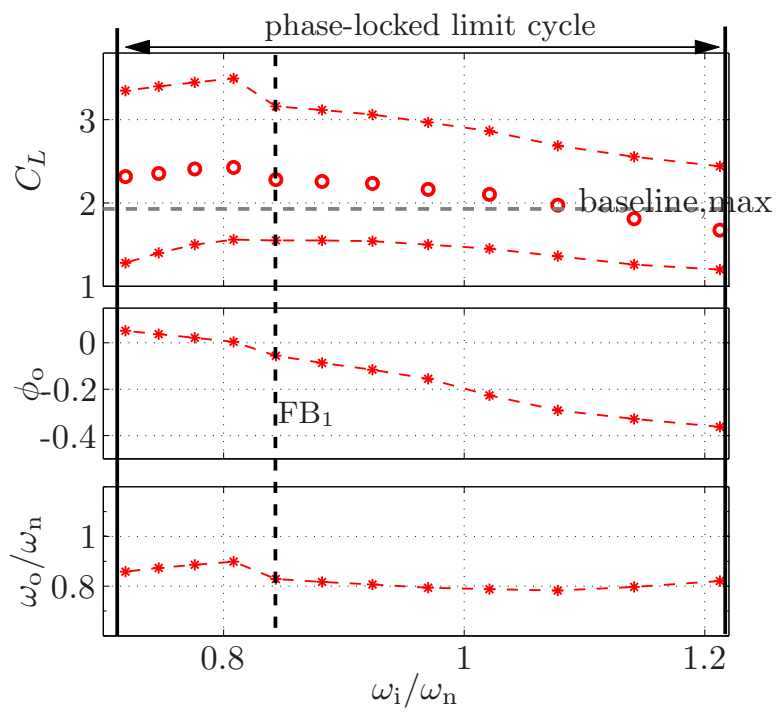

(b) $\alpha=40^{\circ}$

Figure 9. Lift (top), phase between $U_{j}$ and $C_{L}$ (middle), and frequency of $C_{L}$ (bottom) over a range of $\omega_{i}$ that gave phase-locked limit cycle.

At $\alpha=40^{\circ}$, the benefit of the feedback controller is increased. Again, we compare figure 9(b) to the corresponding lift characteristics over various open-loop forcing frequencies in figure 6(c). In figure 6(c), we observe a very small domain of attraction near $\omega_{\mathrm{f}} \approx 0.8$ for the phase-locked limit cycle and the resulting limit cycle has a positive phase shift, $\phi \approx+0.3$. However, the phase-locked limit cycles achieved by this feedback have a wide range of frequencies varying from 0.8 to 0.9 with the corresponding phase shifts ranging from +0.05 to -0.4 . These limit cycles were not achieved by any of the forcing frequencies of the open-loop control in figure $6(\mathrm{c})$. The feedback algorithm again results in phase-locked limit cycles that are not attainable by the open-loop forcing. Also, note that the feedback allows the forcing signals over a range of $0.8 \leq \omega_{\mathrm{i}} / \omega_{\mathrm{n}} \leq 0.9$ to achieve phase-locked limit cycle. This indicates that the feedback results in phase-locked limit cycles for a larger range of forcing frequencies than the open-loop control.

Furthermore in figure 10, we directly compare the lift signal of the two limit cycles: best open-loop case at $\alpha=40^{\circ}$, denoted as $\mathrm{OL}_{1}$ in figure $6(\mathrm{c})$ and the corresponding feedback case, denoted as $\mathrm{FB}_{1}$ in figure 9(b). With open-loop control at fixed $\omega_{\mathrm{f}}$, the flow seems to lock onto the actuation at the higher average lift cycle during earlier periods, with its phase shift closer to $\phi_{\text {best }}$. But after a couple of periods, $\phi$ drifts away from $\phi_{\text {best }}$ and the flow eventually locks onto the lower average lift cycle. On the other hand, the feedback compensator prevents $\phi$ from drifting away and sustains the phase at $\phi_{\text {best }}$ pruducing higher average lift than the open-loop control. Thus, we can conclude that this feedback algorithm stabilizes the limit cycle with a significant lift enhancement that cannot be obtained with the open-loop control.

To insure that the feedback is still required to sustain the achieved phase-locked limit cycle, $\mathrm{FB}_{1}$ is investigated further. Feedback is turned off after the phase-locked limit cycle has been achieved for a long time, and the forcing signal is continued with the open-loop forcing at a fixed frequency, $\omega_{\mathrm{f}}$, as shown in figure 11. Notice that when the forcing signal is continued with the actuation of $\omega_{\mathrm{f}}=\omega_{\mathrm{o}, \mathrm{OL}_{1}}$, the flow drifts back to the previous open-loop limit cycle. When it is continued with actuation oscillating at $\omega_{\mathrm{f}}=\omega_{\mathrm{o}, \mathrm{FB}_{1}}$, the average frequency of the previous feedback output signal, the flow displays a loss of phase-locking to this forcing frequency and it displays a pulling-out phenomenon. These results indicate that the feedback compensator was adjusting its forcing corresponding to the change of output frequency from the flow, and that the feedback is still required to sustain the flow at the high-lift limit cycle. Thus, the feedback achieves high-lift unsteady flow states that cannot be achieved or sustained without it. 


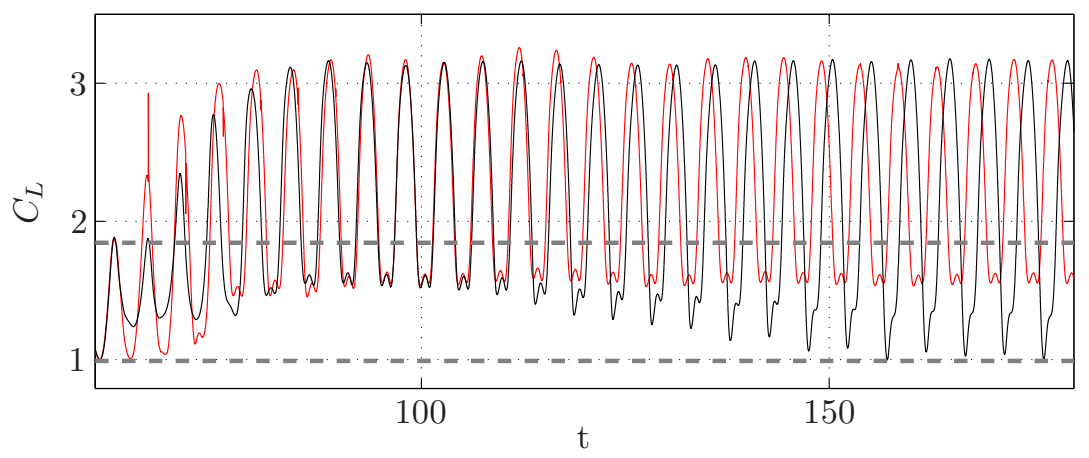

Figure 10. Comparison between open-loop control case (-), that phase locked the flow at the highest average $C_{L}$, and the feedback control case $(-)$. Forcing frequency of this open-loop control is denoted as $\omega_{\mathrm{f}, \mathrm{OL}_{1}}$, and the average output frequency of $C_{L}$ of the feedback control is denoted as $\omega_{\mathrm{o}, \mathrm{FB}_{1}}$.

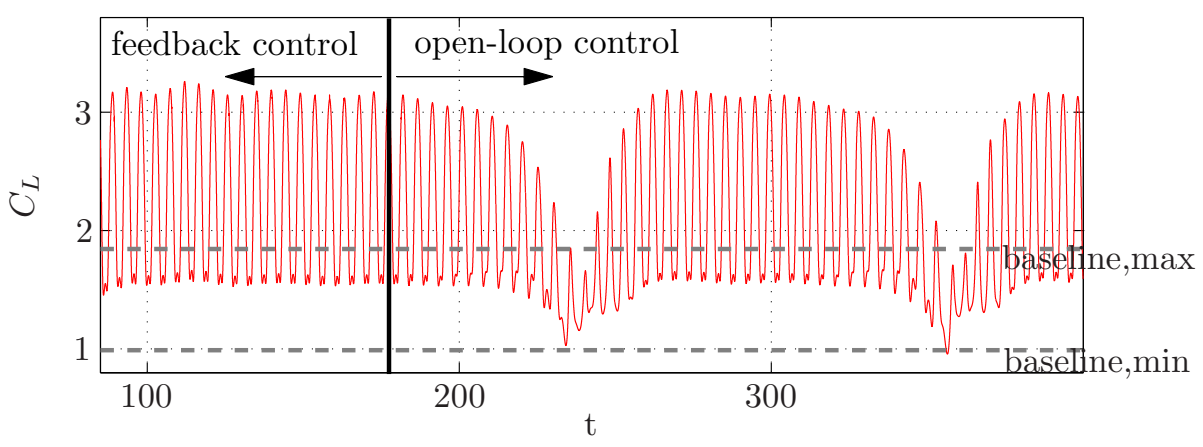

(a) $\omega_{\mathrm{f}}=\omega_{\mathrm{o}, \mathrm{FB}_{1}}$

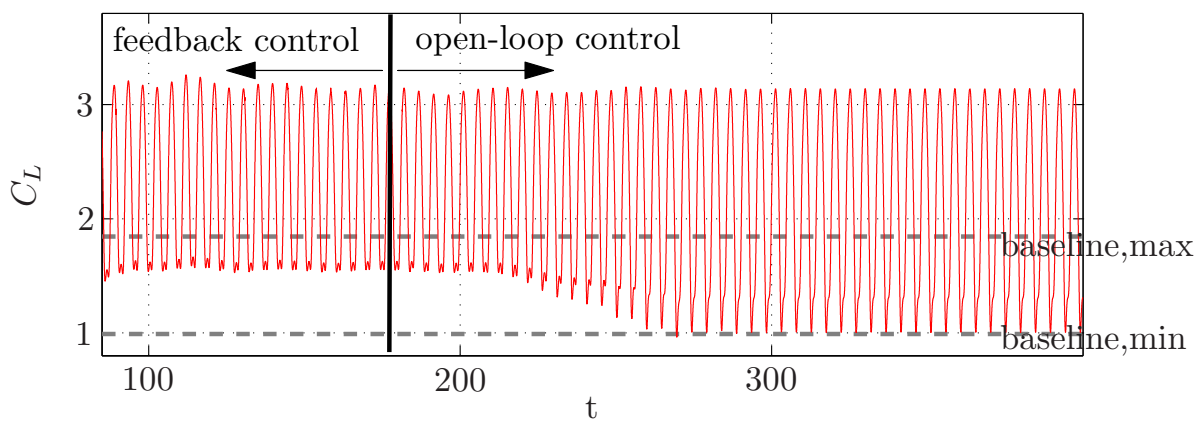

(b) $\omega_{\mathrm{f}}=\omega_{\mathrm{f}, \mathrm{OL}_{1}}$

Figure 11. Continuation of feedback control case in figure 10 with open-loop control of $\omega_{\mathrm{f}}$. 
Recall that when $\omega_{\mathrm{o}}=\omega_{\mathrm{i}}$, we achieve the exact phase shift we ask for, $\phi_{\mathrm{o}}=\phi_{\mathrm{i}}$. This indicates that we can add an integral part to the algorithm to adjust $\omega_{\mathrm{i}}$, such that,

$$
\omega_{\mathrm{i}}^{\mathrm{k}+1}=\omega_{\mathrm{i}}^{\mathrm{k}}+\beta\left(\omega_{\mathrm{o}}^{\mathrm{k}}-\omega_{\mathrm{i}}^{\mathrm{k}}\right) .
$$

We can adjust $\omega_{\mathrm{i}}$ until it reaches $\omega_{\mathrm{o}}$, and thus obtain the exact desired phase shift. Then we would have a robust compensator to explore different limit cycles that are phase locked at various $\phi$ at different $\alpha$. In future investigations, we will explore the phase space with the robust feedback compensator in an attempt to obtain the optimal phase shift as a function of $\alpha$ at different Reynolds numbers.

\section{Summary}

We presented results from a numerical study of unsteady actuation at the leading and trailing edge of a two-dimensional flat plate at high angles of attack at $R e=300$. In general, vortex shedding was amplified when forced at its natural shedding frequency, $\omega_{\mathrm{f}}=\omega_{\mathrm{n}}$, resulting in larger oscillations in lift. However, effective lift enhancement was observed with upstream actuation at the trailing edge. At sufficiently high angles of attack, upstream actuation near the natural shedding frequency excited a subharmonic resonance. Each period within the subharmonic limit cycle was observed to be associated with a particular phase shift between the forcing signal and the lift, and with a particular period-averaged lift. Particularly with upstream actuation at the trailing edge, this period-averaged lift was as high or higher than the maximum lift obtained by the natural shedding cycle, and the phase shift associated with the highest period-averaged lift was denoted as $\phi_{\text {best }}$ at $\alpha=30^{\circ}$ and $40^{\circ}$. Forcing frequencies below $\omega_{\mathrm{n}}$ were also investigated, and it was observed that the open-loop forcing could lead to phase-locked limit cycles at a $\phi$ close to $\phi_{\text {best }}$ resulting in high average lift, but with a decreasing domain of attraction for the phase-locked limit cycles as $\alpha$ increased.

With the goal of obtaining forced limit cycles with the maximum average lift, we designed a feedback algorithm to phase lock the flow at the desired shedding cycle, particularly at $\phi_{\text {best }}$. The control demodulates the lift signal, applies a low-pass filter, adds a phase shift, and finally remodulates, in order to output a sinusoidal forcing with a specified phase shift relative to the lift signal. It was shown that the compensator resulted in the phase-locked limit cycles that the open-loop control could not achieve for $\alpha=30^{\circ}$ and $40^{\circ}$. Particularly for $\alpha=40^{\circ}$, the feedback was able to stabilize the limit cycle that was not stable with any of the open-loop periodic forcing. This results in stable phase-locked limit cycles for a larger range of forcing frequencies than the open-loop control. Also, it was shown that the feedback achieved the high-lift unsteady flow states that open-loop control could not achieve nor sustain even after the states have been achieved for a long period of time.

A companion study by Ahuja et al. ${ }^{20}$ considers a reduced-order model with a balanced proper orthogonal decomposition method to develop feedback control to stabilize LEV over a plate. In the future, we will implement the adjoint-based optimization using the approach of Ahuja et al. to investigate the effectiveness of the feedback algorithm currently presented. Finally, once shown successful, the designed feedback algorithm will be expanded to the vortex formation control behind low-aspect-ratio airfoils in three-dimensional separated flows, that will be in conjunction with the experiments at an oil tunnel facility at Caltech. ${ }^{3}$

\section{References}

\footnotetext{
${ }^{1}$ M. H. Dickinson, K. G. Gotz, "Unsteady Aerodynamic Performance of Model Wings at Low Reynolds Numbers," Journal of Experimental Biology, Vol. 174, pp. 45-64, 1993.

${ }^{2}$ C. P. Ellington, C. van den Berg, A. P. Willmott, A. L. R. Thomas, "Leading-edge Vortices in Insect Flight," Nature, Vol. 384, pp, 626-630, 1996.

${ }^{3}$ T. Colonius, C. W. Rowley, G. Tadmor, D. R. Williams, K. Taira, W. B. Dickson, M. Gharib, and M. Dickinson, "ClosedLoop Control of Leading-Edge and Tip Vortices for Small UAV," Conference on Active Flow Control, DFG, Berlin, Sep. 27-29, 2006.

${ }^{4}$ K. Taira, W. B. Dickson, T. Colonius, M. H. Dickinson, and C. W. Rowley, "Unsteadiness in Flow over a Flat Plate at Angle-of-Attack at Low Reynolds Numbers," 45th Aerospace Sciences Meeting and Exhibit, AIAA, (AIAA 2007-710), Reno, NV, Jan 8-11, 2007.

${ }^{5}$ D. Williams, S. Doshi, and J. Collins, "Control of the Spanwise Distribution of Circulation on NACA 0012 and Flat Plate Wings," 45th Aerospace Sciences Meeting and Exhibit, AIAA, (AIAA 2007-1121), Reno, NV, Jan 8-11, 2007.

${ }^{6}$ S. Ahuja, C. W. Rowley, I. G. Kevrekidis, and M. Wei, "Low-Dimensional Models for Control of Leading-Edge Vortices: Equilibria and Linearized Models," 45th Aerospace Sciences Meeting and Exhibit, AIAA, (AIAA 2007-709), Reno, NV, Jan 8-11, 2007.
} 
${ }^{7}$ A. Glezer and M. Amitay, "Synthetic Jets," Annu. Rev. Fluid Mech., Vol. 34, pp. 503-529, 2002.

${ }^{8}$ D. Greenblatt and I. J. Wygnanski, "The Control of Flow Separation by Periodic Excitation", Progress in Aerospace Sciences, Vol. 36, pp. 487-545, 2000.

${ }^{9}$ M. Amitay, D. R. Smith, V. Kibens, D. E. Parekh, and A. Glezer, "Aerodynamic Flow Control over an Unconventional Airfoil Using Synthetic Jet Actuators," AIAA Journal, Vol. 39, No. 3, 2001.

${ }^{10}$ A. Seifert, D. Greenblatt, and I. J. Wygnanski, "Active Separation Control: an Overview of Reynolds and Mach Numbers Effects," Aerospace Science and Technology, Vol. 8, pp. 569-582, 2004.

${ }^{11} \mathrm{~K}$. Taira and T. Colonius, "The Immersed Boundary Method: A Projection Approach," Journal of Computational Physics, Vol. 225, pp. 2118-2137, 2007.

${ }^{12}$ T. Colonius and K. Taira, "A Fast Immersed Boundary Method Using a Nullspace Approach and Multi-domain Far-field Boundary Conditions," Computer Methods in Applied Mechanics and Engineering, article in press, 2007.

${ }^{13}$ A. Roshko, "Experiments on the Flow Past a Circular Cylinder at Very High Reynolds Number," Journal of Fluid Mechanics, Vol. 10, pp. 345-356, 1961.

${ }^{14}$ P. W. Bearman, "On Vortex Street Wakes," Journal of Fluid Mechanics, Vol. 28, pp.625-641, 1967.

${ }^{15}$ O. M. Griffin, "A Universal Strouhal Number for the 'Locking-on' of Vortex Shedding to the Vibrations of Bluff Cylinders," Journal of Fluid Mechanics, Vol. 85, pp. 591-606, 1978.

${ }^{16}$ M. Amitay and A. Glezer, "Role of Actuation Frequency in Controlled Flow Reattachment over a Stalled Airfoil," AIAA Journal, Vol. 40, No. 2, 2002.

${ }^{17}$ A. Glezer, M. Amitay, and A. M. Honohan, "Aspect of Low- and High-Frequency Actuation for Aerodynamic Flow Control," AIAA Journal, Vol. 43, No. 7, 2005.

${ }^{18}$ J. M. Rullan, P. P. Vlachos, D. P. Telionis, M. D. Zeiger, "Post-Stall Flow Control of Sharp-Edged Wings via Unsteady Blowing," Journal of Aircraft, Vol. 43, No. 6, 2006.

${ }^{19}$ L. Huang, P. G. Huang, R. P. LeBeau, and T. Hauser, "Numerical Study of Blowing and Suction Control Mechanism on NACA0012 Airfoil," Journal of Aircraft, Vol. 41, No. 5, 2004.

${ }^{20}$ S. Ahuja and C. W. Rowley, "Low Dimensional Models for Feedback Stabilization of Unstable Steady States," 46th Aerospace Sciences Meeting and Exhibit, AIAA, (AIAA 2008-553), Reno, NV, Jan 7-10, 2008. 